\title{
ORIGINAL ARTICLE Stability of predictors of mortality after spinal cord injury
}

\author{
JS Krause ${ }^{1}$, LL Saunders ${ }^{1}$ and Y Zhai $^{2}$
}

Study design: Cohort study.

Objectives: To identify the stability of socio-environmental, behavioral and health predictors of mortality over an 8-year time frame. Setting: Data were analyzed at a large medical university in the Southeast United States of America (USA).

Methods: Adults with residual impairment from a spinal cord injury (SCI) who were at least 1-year post-injury at assessment were recruited through a large specialty hospital in the Southeast USA. A total of 1209 participants were included in the final analysis. A piecewise exponential model with two equal time intervals ( 8 years total) was used to assess the stability of the hazard and the predictors over time.

Results: The hazard did significantly change over time, where the hazard in the first time interval was significantly lower than the second. There were no interactions between the socio-environmental, behavior or health factors and time, although there was a significant interaction between age at injury (a demographic variable) and time.

Conclusion: These results suggest there is stability in the association between the predictors and mortality, even over an 8-year time period. Results reinforce the use of historic variables for prediction of mortality in persons with $\mathrm{SCl}$.

Spinal Cord (2012) 50, 281-284; doi:10.1038/sc.2011.158; published online 10 January 2012

Keywords: spinal cord injury; mortality; proportional hazards models

\section{INTRODUCTION}

It has been well established that spinal cord injury (SCI) is associated with greater hazard of mortality; the extent to which depends largely upon the nature and severity of the injury. ${ }^{1,2}$ Mortality is highest among those who are ventilator dependent. ${ }^{3}$ It is lowest among those with neurologically incomplete injuries. Among those who do not have neurologically incomplete injuries, the hazard of mortality is related to the neurological level of injury, with those who have higher levels having greater hazard of mortality.

More recent research has investigated a wider array of predictive factors, beyond demographic and injury characteristics, in relation to mortality. Krause ${ }^{4}$ developed a theoretical risk model that distinguishes between several sets of predictive factors and their relative importance to mortality. In a series of investigations, each of four sets of predictor variables was related to a greater hazard of mortality including psychological, socio-environmental, behavioral and health factors. ${ }^{5-8}$ Seven individual predictive factors were identified in an overall test of the theoretical risk model with the predictors assessed $\sim 8$ years before determination of mortality status. ${ }^{9}$ These significant predictors included four health status variables (surgeries to repair pressure ulcers, history of amputation/fracture, probable major depression and days hospitalized in the year before assessment), two behavioral predictors (psychotropic prescription medication use, binge drinking) and one socio-environmental predictor (low income).

Some methodological challenges in studies of mortality relate to sample size, stability of the predictor variables and the interval between assessment of predictors and determination of mortality status. Mortality occurs at a relatively low rate, so large participant samples are required to identify hazard of mortality. SCI is a relatively rare event, and identifying sufficient numbers of participants can be problematic. The longer interval length between assessment and determination of mortality status is typically required to allow for a sufficient number of cases of mortality, yet the predictors themselves may lose some of their potency over time. For instance, in the aforementioned study, ${ }^{9}$ variables such as hospitalized days within the past year or a depressive diagnosis may change over time, more so than other variables that are historic in nature (for example, history of amputation). It is therefore important to evaluate the extent to which these types of predictors lose their potency in predicting future mortality as the length of time between assessment and determination of mortality status increases. To date, we could find no literature addressing this issue with SCI.

\section{Purpose}

Our purpose was to identify the stability of the seven non-demographic- and non-injury-related predictors of mortality identified by Krause et al. ${ }^{9}$ with mortality over time. Identifying the stability of predictors allows us to determine the predictive limits of these variables and the relative stability of different variables within these classes of predictor variables. Our hypothesis is that the hazard ratio for any given predictor will be inversely related to the time elapsed between assessment of the predictor and determination of mortality status.

\section{MATERIALS AND METHODS \\ Participants}

After obtaining approval from the Institutional Review Board, potential participants were identified from a specialty hospital in the Southeast United

\footnotetext{
${ }^{1}$ Department of Health Sciences and Research, College of Health Professions, Medical University of South Carolina, Charleston, SC, USA and ${ }^{2}$ Epidemiology and Surveillance Research Department, American Cancer Society, Atlanta, GA, USA

Correspondence: Dr JS Krause, Department of Health Sciences and Research, College of Health Professions, Medical University of South Carolina, 77 President St, Suite C101, MSC 700, Charleston, SC 29425. USA.

E-mail: krause@musc.edu
}

Received 19 May 2011; revised 17 November 2011; accepted 20 November 2011; published online 10 January 2012 
States of America. All adults with traumatic SCI who were at least 1-year postinjury and had at least some residual impairment were enrolled in the study. A total of 1386 participated ( $72 \%$ response rate). Characteristics of this cohort are documented in more detail elsewhere. ${ }^{9}$ Complete information was not available for 177 participants; therefore, they were excluded from the analysis.

\section{Procedures}

Data were collected through survey between July 1997 and June 1998. Preliminary cover letters were sent to explain the study and to alert potential participants that materials would be forthcoming. Approximately 4-6 weeks later, materials were sent to participants. Two mailings were used for nonrespondents as well as a follow-up phone call. All materials were scanned for missing information, and participants were contacted in the event of large amounts of missing variables (for example, pages sticking together and not completed). Participants were allowed to skip any items they felt were sensitive or personal. Respondents received \$20 in remuneration, and they were made eligible for $\$ 1500$ in drawings. All data were entered and verified to eliminate errors. Mortality status was evaluated using the National Death Index as of 31 December 2005 (a timeframe consistent with that in the original research).

\section{Measures}

A composite of several individual measures was developed for the study. It includes components from the theoretical risk model including: demographic and injury characteristics, environmental, behavioral and health factors. For the purpose of this manuscript, several variables were utilized including age, gender, race-ethnicity, years since injury and injury severity. Injury severity was classified into five groups based on a combination of injury level and ambulatory status. Ambulatory participants were classified as either cervical or non-cervical, with non-cervical ambulatory serving as the reference group. Non-ambulatory participants were grouped according to injury level as follows: C1-C4, C5-C8 and non-cervical.

Two questions were used from the Behavioral Risk Factor Surveillance System. ${ }^{10}$ Poverty was assessed using a standardized Behavioral Risk Factor Surveillance System item regarding family income with a cutoff of $\$ 20000$ (higher income was reference group). Binge drinking was defined by the number of occasions within the past month drinking five or more drinks.

A psychotropic prescription medication use composite score was based on the use of medications to treat four conditions (sleep, spasticity, pain and depression). Participants were asked the number of surgeries they have had since SCI onset to repair pressure ulcers. They were also asked whether they have ever had a fracture or amputation since their SCI.

Probable major depression was measured with the Older Adult Health and Mood Questionnaire, a 22 item measure developed specifically for those with health concerns or disabling conditions. ${ }^{11}$ This measure has been widely used with SCI. ${ }^{12,13}$ A score of 11 or greater was used to classify probable major depression. A more complete description of these measures appears elsewhere. ${ }^{9}$

\section{Analysis}

First, we used a piecewise exponential model to assess if the hazard changes over time. Two equal 4-year intervals were created, and the hazard was assessed over these two intervals. The second (last) interval was used as the reference. Along with the time interval variable, the other independent variables (injury severity, race, gender, age at onset, years post-injury, income, prescription medication use, binge drinking, days in the hospital, fracture/amputation, sore surgeries and depression) were also included. Next, another piecewise exponential model was created, and interactions between the time interval and the independent variables were included to assess the change in coefficients across the time intervals. A significant interaction would indicate a predictor was more highly related with mortality during one of the two intervals. Mortality was assessed as of 31 December 2005. Persons who were not determined to be deceased at that time were assumed to be alive. Independent variables were all measured between 1997 and 1998.

\section{Statement of ethics}

We certify that all applicable institutional and governmental regulations concerning the ethical use of human volunteers were followed during the course of this research.

\section{RESULTS}

Our first piecewise exponential model included a time interval variable along with the other independent variables. Results of this model showed that the hazard was not constant over time $(P=0.0001)$. The hazard of mortality in the first time interval was significantly lower than in the second (estimate $=-0.5549$ ).

In the second analysis, we included interaction terms between the time interval variable and each independent variable (Table 1). The only significant interaction was between time and age at onset $(P=0.0226)$. All other variables showed no significant interaction with the time interval, signifying that those parameter estimates did not significantly change over time and were removed from the model.

The significant predictors of mortality included injury severity, age and years since injury. Of the non-biographic and injury characteristics, poverty, psychotropic prescription medication use, amputation/ fracture, pressure sore surgeries and major depression all were statistically significant.

\section{DISCUSSION}

The results of this study suggest there is considerable stability in the relationship between socio-environmental, behavioral and health predictors with the hazard of mortality, even when predictor variables were assessed up to 8 years before the determination of mortality status. This is clearly indicated by the absence of significant interaction effects by time, as the only significant interaction was for age at injury onset. Although function may change somewhat over time in some cases, our focus is only on the aforementioned variables and not injury or functional status. This relationship held, even when the predictors would, by their nature, be likely to change over time (for example, depressive disorder).

There are several possible explanations for these findings. First, some health-related variables clearly reflect historical events, such as amputations or surgeries for pressure ulcers. Although these may change, in that additional people may have amputations and the number of surgeries for pressure ulcers may increase, the core events are historical. Therefore, no change would be expected over time, other than that related to new events. Other variables may be more stable than is readily apparent. For instance, although the number of hospitalizations from year-to-year will vary depending on underlying conditions, individuals prone to hospitalization at one time are certainly more likely to be re-hospitalized. ${ }^{14}$ Also, the underlying conditions leading to hospitalization may persist, such that the individual has some change in health status. Similarly, although depressive disorders were measured at a single point in time, those who have a history of a depressive disorder at one time are also more likely to have a depressive disorder at a later point in time. ${ }^{15}$ Therefore, all health-related variables have some degree of stability, although we cannot determine the extent to which the predictors themselves may change over time from our current data.

Other predictors that reflect non-health variables, including poverty and two behavioral predictors, also may change over time. Because we cannot determine the amount of actual change, we are unable to determine whether time-dependent covariates that update status of these variables would ultimately prove to be better predictors. However, because there is no interaction between the predictor or outcome over time, there must be some underlying characteristics that are being measured by these predictors.

Results from this model were mostly consistent with the original article, ${ }^{9}$ but there were a few differences. After adding the time and time $\times$ age at injury variables, days in the hospital and binge drinking 
Table 1 Piecewise exponential model of mortality using two equal time intervals

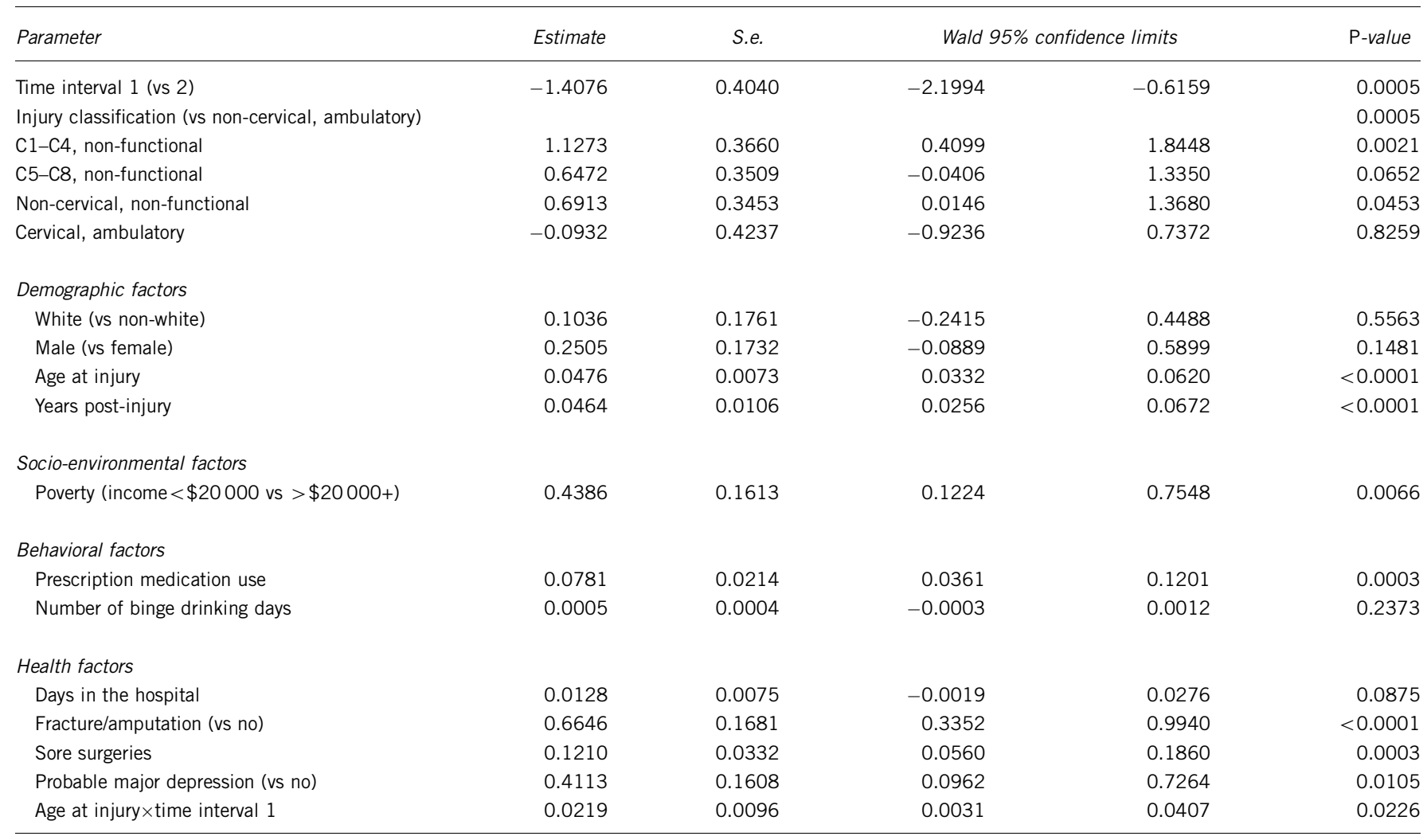

were no longer statistically significant. It is important to continue evaluating risk of mortality to establish the appropriate strategies to intervene at policy, behavioral and health outcome levels.

\section{Limitations}

There were several study limitations. First, the absence of data timedependent covariates precludes assessment of the stability of the predictors themselves. That in no way negates the current findings, but it would be interesting to determine the extent of change in the predictors over time. Second, we were limited to two time intervals0-4 and 5-8 years. We cannot determine whether the stability will be more or less over longer periods of time. Because this is an ongoing study, additional analyses will be conducted in future years as data become available. Third, we used self-report for all variables. Reporting of most variables was straightforward, so this is not likely problematic. Nevertheless, any errors related to self-report bias would weaken the associations between the predictors and mortality (that is, the true relationship might be stronger). Fourth, the sample size, although large for this diversity of variables with a specialty population, still is relatively small in terms of overall mortality. Last, the number of deaths increased in each model, which could affect variables reaching statistical significance.

\section{Future research}

Future research would benefit from investigation of a wider array of predictive factors, testing their relationships with mortality over varying intervals. Following participants over a long period of time would increase the proportion of deceased cases. The addition of more participants would increase the power of this research. Continued research is needed before we will gain a more comprehensive understanding of predictors of mortality after SCI and have the ability to successfully intervene to promote greater longevity. Future analysis may also benefit from the application of time-dependent analytic techniques. Further research is needed to enhance our ability to predict and ultimately delay mortality.

\section{CONFLICT OF INTEREST}

The authors declare no conflict of interest.

\section{ACKNOWLEDGEMENTS}

The contents of the publication were developed under a grant from the Department of Education, NIDRR grant numbers H133G050165 and H133B090005, and the NIH grant numbers 1R01 NS 48117 and 1R01 HD37496. However, those contents do not necessarily represent the policy of the Department of Education or the NIH, and you should not assume endorsement by the Federal Government.

1 DeVivo MJ, Krause JS, Lammertse DP. Recent trends in mortality and causes of death among persons with spinal cord injury. Arch Phys Med Rehabil 1999; 80: 1411-1419.

2 Krause JS, DeVivo MJ, Jackson AB. Health status, community integration, and economic risk factors for mortality after spinal cord injury. Arch Phys Med Rehabil 2004; 85: 1764-1773.

3 Shavelle RM, DeVivo MJ, Strauss DJ, Paculdo DR, Lammertse DP, Day SM. Long-term survival of persons ventilator dependent after spinal cord injury. J Spinal Cord Med 2006; 29: 511-519.

4 Krause JS. Secondary conditions and spinal cord injury: a model for prediction and prevention. Top Spinal Cord Inj Rehabil 1996; 2: 217-227.

5 Krause JS, Carter R, Zhai Y, Reed K. Psychologic factors and risk of mortality after spinal cord injury. Arch Phys Med Rehabil 2009; 90: 628-633. 
6 Krause JS, Carter RE. Risk of mortality after spinal cord injury: relationship with social support, education, and income. Spinal Cord 2009; 47: 592-596.

7 Krause JS, Carter RE, Pickelsimer E. Behavioral risk factors of mortality after spinal cord injury. Arch Phys Med Rehabil 2009; 90: 95-101.

8 Krause JS, Carter RE, Pickelsimer E, Wilson D. A prospective study of health and risk of mortality after spinal cord injury. Arch Phys Med Rehabil 2008; 89: 1482-1491.

9 Krause JS, Zhai Y, Saunders LL, Carter RE. Risk of mortality after spinal cord injury: an 8-year prospective study. Arch Phys Med Rehabil 2009; 90: 1708-1715.

10 Centers for Disease Control. Behavioral Risk Factor Questionnaire 1996. Retrieved from http://www.cdc.gov/brfss/questionnaires/pdf-ques/96brfss.pdf.

11 Kemp BJ, Adams BM. The older adult health and mood questionnaire: a measure of geriatric depressive disorder. J Ger Psychiat Neurol 1995; 8: 162-167.
12 Krause JS, Kemp B, Coker JL. Depression after spinal cord injury: relation to gender, ethnicity, aging, and socioeconomic indicators. Arch Phys Med Rehabil 2000; 81: 1099-1109.

13 Kahan JS, Mitchell JM, Kemp BJ, Adkins RH. The results of a 6-month treatment for depression on symptoms, life satisfaction, and community activities among individuals aging with a disability. Rehabil Psychol 2006; 51: 13-22.

14 Charlifue S, Lammertse D, Adkins R. Aging with spinal cord injury: changes in selected health indices and life satisfaction. Arch Phys Med Rehabil 2004; 85: 1848-1853.

15 Hoffman JM, Bombardier CH, Graves DE, Kalpakjian CZ, Krause JS. A longitudinal study of depression from one to five years after spinal cord injury. Arch Phys Med Rehabil 2011; 92: 411-418. 